\title{
Glaucoma after Ocular Surgery or Trauma
}

\section{The Role of Infiltrating Monocytes and Their Response to Cytokine Inhibitors}

\author{
Xiaoniao Chen, ${ }^{*}{ }^{\dagger}$ Fengyang Lei, ${ }^{*}$ Chengxin Zhou, ${ }^{*}$ James Chodosh, ${ }^{*}$ Liqiang Wang, ${ }^{\dagger}$ Yifei Huang ${ }^{\dagger}$ Claes H. Dohlman, ${ }^{*}$ and \\ Eleftherios I. Paschalis*
}

From the Boston Keratoprosthesis Laboratory, ${ }^{*}$ Department of Ophthalmology, Massachusetts Eye and Ear, Harvard Medical School, Boston, Massachusetts; and the Department of Ophthalmology, ${ }^{\dagger}$ People's Liberation Army General Hospital, Beijing, China

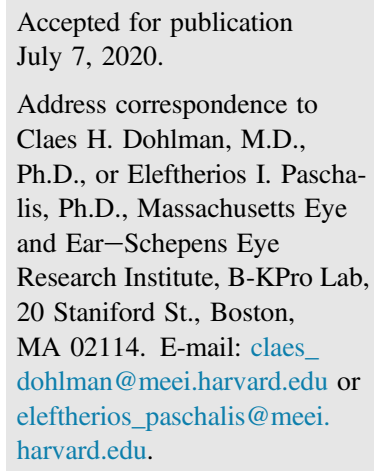

\begin{abstract}
Glaucoma is a frequent and devastating long-term complication following ocular trauma, including corneal surgery, open globe injury, chemical burn, and infection. Postevent inflammation and neuroglial remodeling play a key role in subsequent ganglion cell apoptosis and glaucoma. To this end, this study was designed to investigate the amplifying role of monocyte infiltration into the retina. By using three different ocular injury mouse models (corneal suture, penetrating keratoplasty, and globe injury) and monocyte fate mapping techniques, we show that ocular trauma or surgery can cause robust infiltration of bone marrow-derived monocytes into the retina and subsequent neuroinflammation by up-regulation of Tnf, Il1b, and Il6 mRNA within 24 hours. This is accompanied by ganglion cell apoptosis and neurodegeneration. Prompt inhibition of tumor necrosis factor- $\alpha$ or IL-1 $\beta$ markedly suppresses monocyte infiltration and ganglion cell loss. Thus, acute ocular injury (surgical or trauma) can lead to rapid neuroretinal inflammation and subsequent ganglion cell loss, the hallmark of glaucoma. Infiltrating monocytes play a central role in this process, likely amplifying the inflammatory cascade, aiding in the activation of retinal microglia. Prompt administration of cytokine inhibitors after ocular injury prevents this infiltration and ameliorates the damage to the retina-suggesting that it may be used prophylactically for neuroprotection against post-traumatic glaucoma. (Am J Pathol 2020, 190: 2056-2066; https://doi.org/10.1016/j.ajpath.2020.07.006)
\end{abstract}

Glaucoma is a frequent and devastating long-term complication after acute ocular trauma, including corneal surgery, open globe injury, chemical burn, and infection. ${ }^{1-17}$ Intraocular pressure (IOP) elevation in the healing stage has usually been considered the primary reason for posttraumatic glaucoma; however, recent animal studies have demonstrated that inflammation might also be an important, IOP-independent pathway, justifying further investigation. ${ }^{18-22}$ At the molecular level, tumor necrosis factor$\alpha$ (TNF- $\alpha$ ) has been shown to be a key mediator of such neuroinflammation, and its prompt inhibition using a monoclonal antibody has been markedly protective against ganglion cell damage - the hallmark of glaucoma. ${ }^{18,20,23-28}$ TNF- $\alpha$ up-regulation has also been found in the blood of patients implanted with an artificial cornea (Boston
Keratoprosthesis), even years after the surgery, which may be a factor in the progression of glaucoma despite apparently normal IOP in keratoprosthesis recipients. ${ }^{29}$ In summary, these data suggest that targeted immunomodulation should be able to reduce the severity of late glaucoma following acute injury to the eye.

Supported by the Boston Keratoprosthesis Research Fund, Massachusetts Eye and Ear (X.C., F.L., C.Z., J.C., C.H.D., E.I.P.), the Eleanor and Miles Shore Fund (E.I.P.), the Massachusetts Lions Eye Research Fund (E.I.P.), NIH National Eye Institute core grant P30EY003790, R01 grant 5R01EY029437-03 (E.I.P). the National Key R\&D Program of China Project 2017YFA0103204 (L.W. and X.C.)

Disclosures: None declared. 
The present study was designed to further explore the development of post-traumatic glaucoma and particularly to investigate a possible amplifying pathway, effectuated by infiltration of bone marrow-derived monocytes into the retina. This is important because it was recently shown that monocytes can contribute to sustained neurodegeneration of the retina, long after chemical injury. ${ }^{30-34}$

\section{Materials and Methods}

Animals

All animal-based procedures were performed in accordance with the Association for Research in Vision and Ophthalmology Statement for the Use of Animals in Ophthalmic and Vision Research and the NIH's Guide for the Care and Use of Laboratory Animals. ${ }^{35}$ Animal Care Committee protocol approval was obtained at the Massachusetts Eye and Ear (Boston, MA). The 6to 8-week-old (body weight, approximately $25 \mathrm{~g}$ ) C57BL/6J, $C \times 3 \mathrm{crl}^{\mathrm{EGFP} / \mathrm{EGFP}}$, and B6. Thy $1^{\mathrm{YPP}}$ mice were obtained from the Jackson Laboratory (Bar Harbor, ME). $C x 3 c r 1^{+ \text {EGFP }}$ mice were generated by mating C57BL/6J with $C x 3 c r 1^{\text {EGFP/EGFP }}$. Bone marrow chimeras were generated, as previously described. ${ }^{31}$ Briefly, C57BL/6J mice were myelodepleted with three i.p. injections of busulfan (Sigma-Aldrich, St Louis, MO) (35 mg/kg) administered 7, 5 , and 3 days before bone marrow transfer. Bone marrow cells $\left(5 \times 10^{6}\right.$ total $)$ from $C \times 3 c{ }^{+/ E G F P}$ mice were transferred to the myelodepleted C57BL/6J mice through tail vein injection 1 month before corneal injury. Bactrim was given ad lib for 15 days after myelodepletion to avoid infection.

The mouse strain C57BL/6J is a well-characterized wildtype black mouse, used in most of studies in immune studies. The $C x 3 \mathrm{crl}^{+/ \text {EGFP }}$ reporter mouse is based on a BL/6 wildtype background and expresses green fluorescent protein under the fractalkine receptor $\mathrm{Cx} 3 \mathrm{crl}$ gene promoter. This labels mature monocytes/macrophages and microglia, and is commonly used in neuroglia biology. The Thyl ${ }^{\mathrm{YFP}}$ mouse is also a BL/6 background mouse strain, genetically modified to express yellow fluorescent protein under the Thyl gene promoter. Thyl is expressing in retinal ganglion cells and nerve axons. Bone marrow $C x 3 \mathrm{crl}^{+/ \text {EGFP }}$ mice were produced to trace and distinguish peripheral monocytes/macrophages from yolk sac-derived microglia.

\section{Corneal Suture Model}

A single 11-0 nylon suture (2881 G: 11-0 Ethilon black 5" BV50-3 taper; Johnson \& Johnson, Neenah, WI) was placed full thickness in the cornea. Topical Polytrim antibiotic (Bausch \& Lomb Inc., Bridgewater, NJ) was applied after the procedure, followed by tarsorrhaphy using 8-0 nylon sutures (Sharpoint; Surgical Specialties Co., Wyomissing, PA). Immediately after the procedure, mice received s.c. buprenorphine injection every 12 hours $(0.1 \mathrm{mg} / \mathrm{kg}$; Indivior, North Chesterfield, VA) for 3 days after surgery. Eyes were analyzed at 24 hours and 7 days after the injury.

\section{Autologous Penetrating Keratoplasty Model}

All surgeries were performed in anesthetized mice using a surgical microscope (S7; Zeiss, Oberkochen, Germany). A drop of $0.5 \%$ phenylephrine hydrochloride (Altaire Pharmaceuticals, New York, NY) was applied for pupil dilation 15 minutes before the procedure. Corneas were marked with a 2-mm-diameter biopsy punch (Miltex, Plainsboro, NJ). Viscoelastic (Viscoat; Alcon, Irvine, CA) was injected through a groove with a 30-gauge cannula (Rumex, Clearwater, FL) to maintain a deep anterior chamber. Corneas were trephined and then excised using Vannas scissors (Storz Instruments Company, San Damis, CA), and immediately resutured back to the same eye using 11-0 nylon sutures (Sharpoint; Angiotech Pharmaceuticals, Vancouver, BC, Canada) in interrupted manner. Topical Polytrim (Allergan, Madison, NJ) antibiotic was applied after the procedure, followed by tarsorrhaphy using 8-0 nylon sutures. Immediately after the procedure, mice received s.c. buprenorphine injection every 12 hours for 3 days after surgery. Eyes were analyzed at 24 hours and 7 days after the injury.

\section{Penetrating Keratoplasty with Globe Trauma Model}

Controlled globe trauma was performed surgically by penetrating the cornea using a 2-mm trephine and extending the injury to the iris with iridotomy and to the lens with mechanical lens removal (forceps) (Katena, Denville, NJ). The cornea was then immediately resutured using 110 nylon sutures, and topical Polytrim antibiotic was administered to the eye, followed by tarsorrhaphy with 80 nylon sutures. Eyes were analyzed at 24 hours and 7 days. Immediately after the procedure, mice received s.c. buprenorphine injection every 12 hours for 3 days after surgery.

\section{Recombinant IL-1 $\beta$ Injection into the Anterior Chamber}

Anterior chamber tap and inject of $1 \mu \mathrm{L}$ of recombinant IL-1 $\beta$ (1 $\mu \mathrm{g}$; catalog number 211-11B; PeproTech, Cranbury, NJ) was performed using a 33-gauge needle in BMT $C x 3 \mathrm{crl}^{+/ \text {EGFP }}$ reporter mice. Eyes were treated with topical Polytrim antibiotic and enucleated 24 hours later for retinal analysis using real-time quantitative RT-PCR and confocal microscopy.

\section{Systemic Administration of Pharmaceutical Agents}

Dexamethasone, $20 \mathrm{mg} / \mathrm{kg}$ (Baxter, Deerfield, IL), infliximab, $10 \mathrm{mg} / \mathrm{kg}$ (Remicade; Janssen Biotech, Inc., Horsham, PA), anakinra, $10 \mathrm{mg} / \mathrm{kg}$ (Kineret; Sobi, Inc., Waltham, MA), and a combination of infliximab (10 $\mathrm{mg} / \mathrm{kg})+$ anakinra $(10 \mathrm{mg} / \mathrm{kg})$ were injected intraperitoneally immediately after the procedure. For control, IgG (10 or $20 \mathrm{mg} / \mathrm{kg}$; catalog number 500-M00; PeproTech, Rocky Hill, NJ) was injected intraperitoneally. 

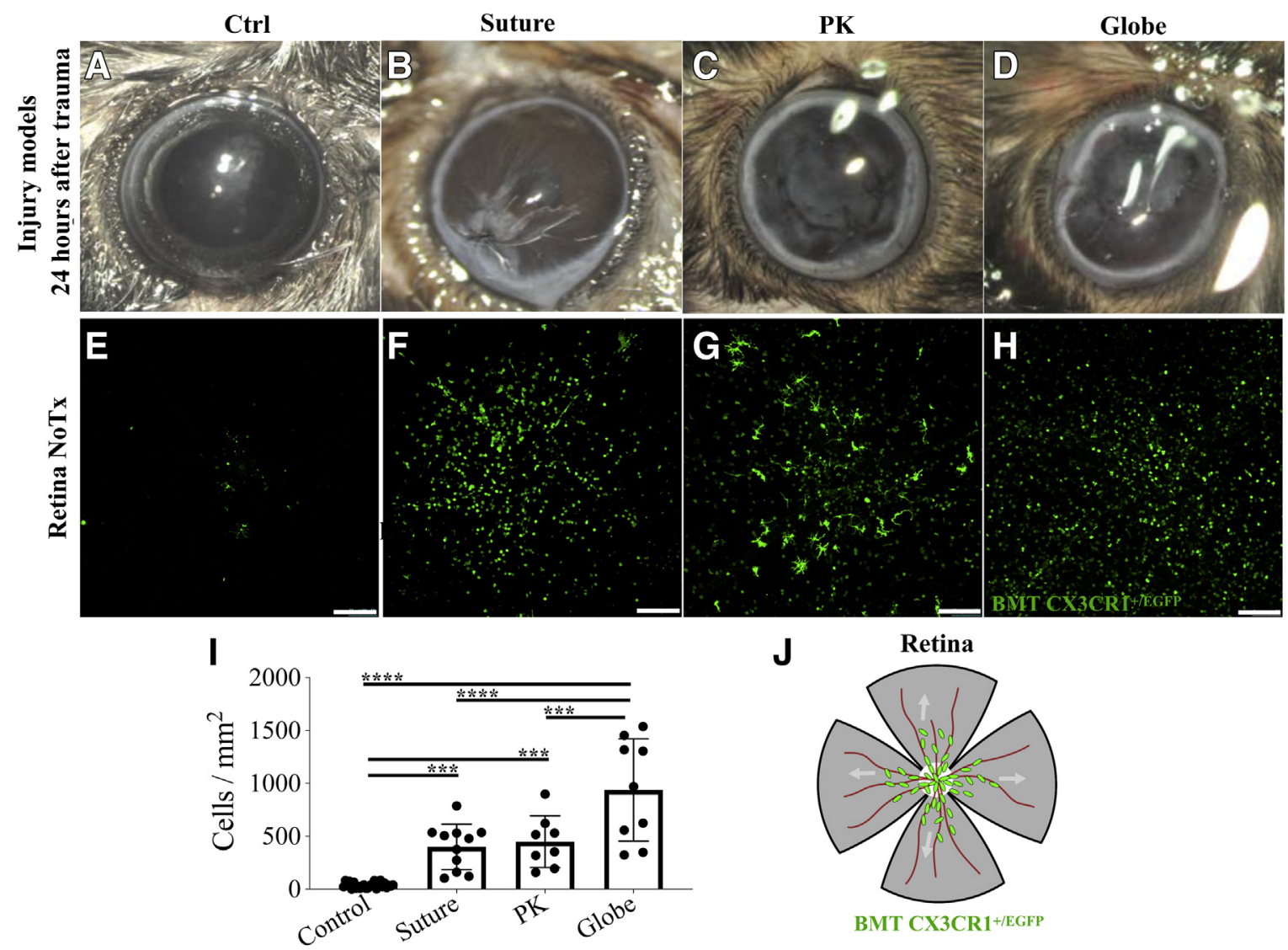

K

$\mathrm{CX3CR1}{ }^{+}$cell in the retina after $\mathrm{PK}$
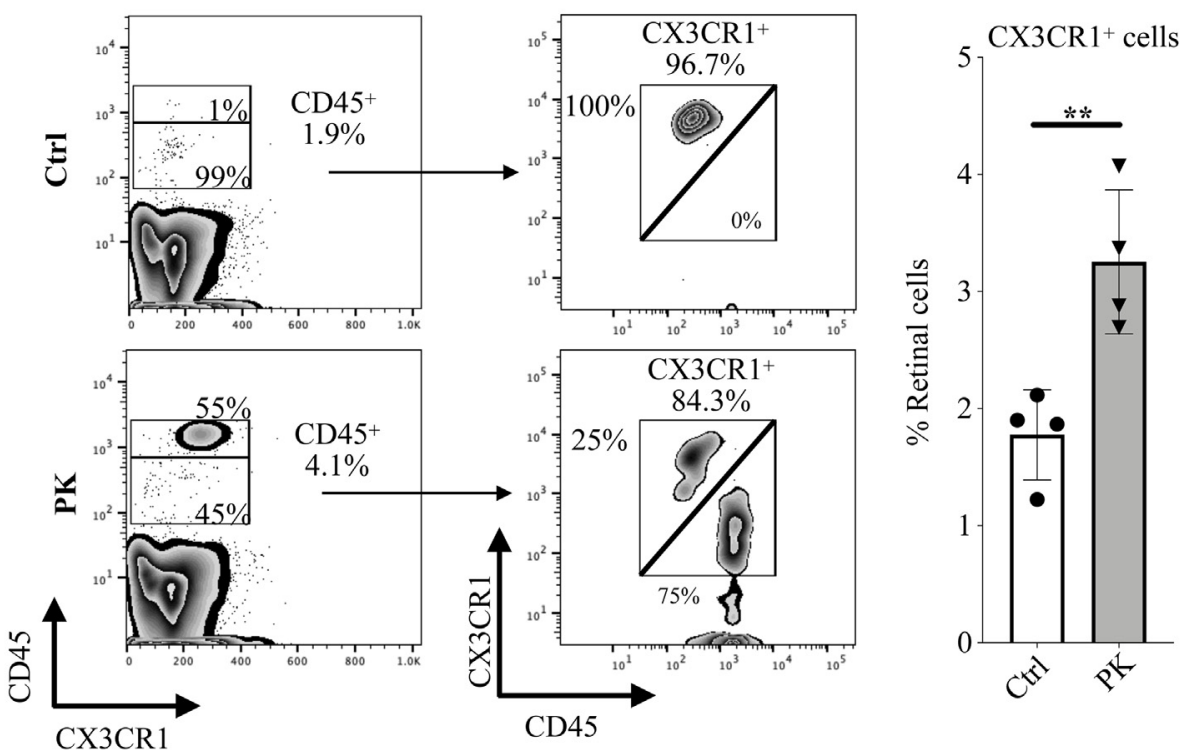

Figure 1 0cular surgery or trauma causes rapid infiltration of bone marrow-derived C-X3-C motif chemokine receptor $1-$ positive $\left(C X 3 C R 1^{+}\right)$monocytes into the retina. A-D: Clinical presentation of mouse eyes with no injury $(\mathbf{A})$, penetrating corneal suture injury $(\mathbf{B})$, penetrating keratoplasty $(\mathrm{PK} ; \mathbf{C})$, and PK with globe trauma (Globe; D) at 24 hours. E-H: Flat mount retinal confocal images of BL6;CX3cr1 ${ }^{+/ E G F P}$ chimeras infiltrated by bone marrow-derived CX3CR1 ${ }^{+}$monocytes within 24 hours of the injury. I: Quantification of the relative number of infiltrated CX3CR1 ${ }^{+/ E G F P}$ monocytes showing significant increase following injury. J: Schematic representation of flat mount retinas, showing the optic nerve (central white circle), the retinal vessels (red lines), and the infiltrating CX3CR1 ${ }^{+}$ monocytes (green oval). K: Flow cytometry of retinas from $\mathrm{C} \times 3 \mathrm{Cr} 1^{+/ \mathrm{EGFP}}$ reporter mice. Retinal monocytes/macrophages were selected using the $\mathrm{CX} 3 \mathrm{CR} 1^{+}$and $\mathrm{CD}_{4} 5^{+}$markers. Two monocyte/macrophage subpopulations were identified, the CD45 hi (peripheral monocytes) and CD45 ${ }^{\text {lo }}$ (microglia). Further analysis confirms the infiltration of $C X 3 C R 1^{10}$ (peripheral monocytes) after surgical injury, whereas the CX3CR1 ${ }^{\text {hi }}$ microglia number remains unchanged. I: Analysis of variance analysis with Tukey correction for multiple comparison. K: Unpaired $t$-test. $n \geq 8$ mice per group $(\mathbf{I}) ; n=4$ mice per group $(\mathbf{K})$. ${ }^{* *} P<0.01,{ }^{* *} P<0.001$, and $* * * * P<0.0001$. Scale bars $=100 \mu \mathrm{m}(\mathbf{E}-\mathbf{H})$. Ctrl, naive control; EGFP, enhanced green fluorescent protein; NoTx, no treatment control. 


\section{BMT CX3CR1 ${ }^{+/ E G F P}$ cell infiltration after PK}
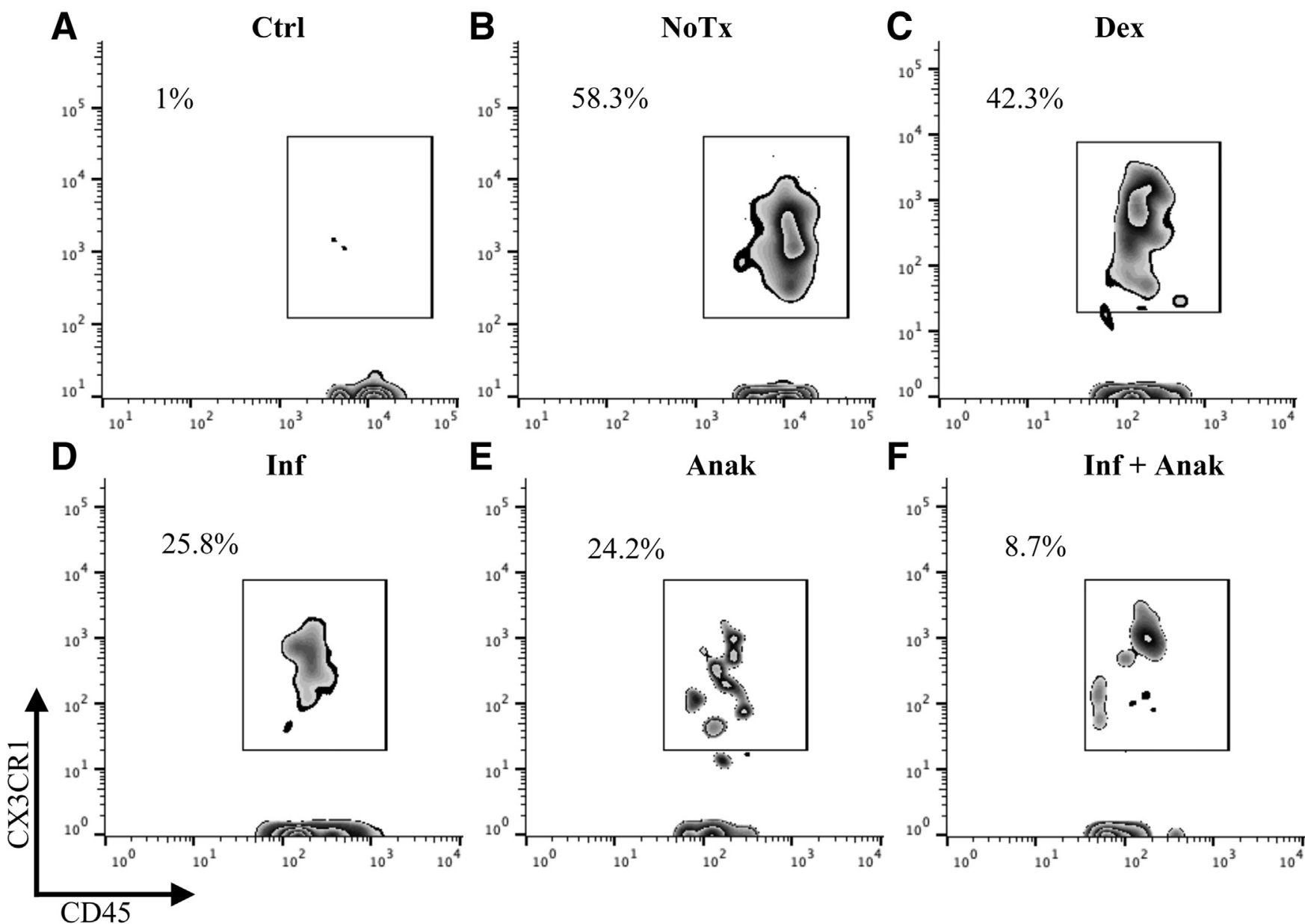

E

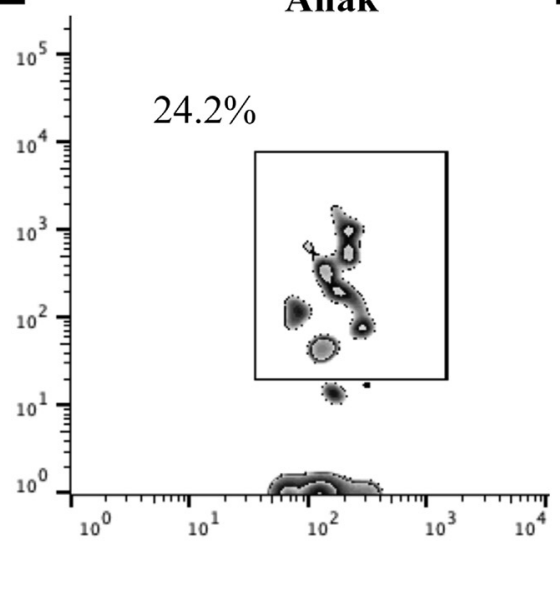

$\mathbf{F}$

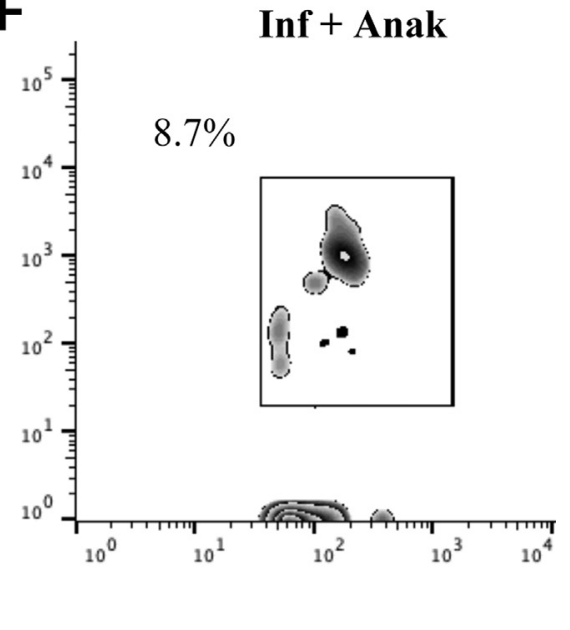

G BMT CX3CR1 ${ }^{+/ E G F P}$ cell infiltration after PK

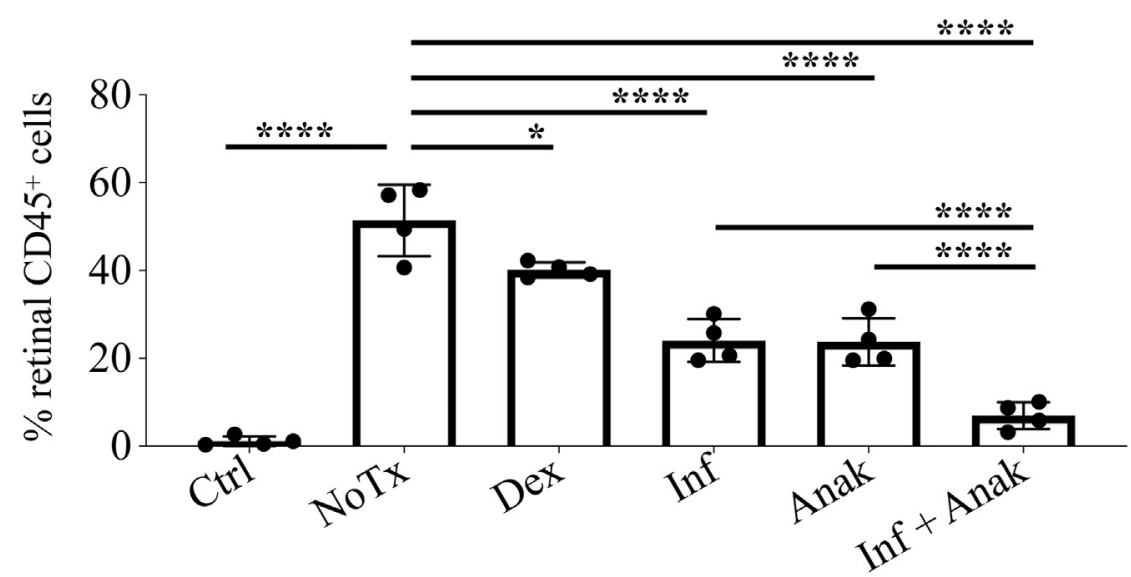

Figure 2 Tumor necrosis factor- $\alpha$ (TNF- $\alpha$ ) or IL-1 $\beta$ inhibition prevents $C D 45^{+}$myeloid cell infiltration into the retina after ocular surgical injury. A-G: Flow cytometry (A-F) and quantification (G) of the relative number of C-X3-C motif chemokine receptor 1-positive (CX3CR1 ${ }^{+/ E G F P}$ ) infiltrating monocytes into the retina at 24 hours after penetrating keratoplasty (PK) injury. A and G: PK injury causes marked infiltration of CX3CR1 ${ }^{+}$monocytes into the retina within 24 hours. B and G: Systemic administration of corticosteroids [dexamethasone (Dex)] has minimal effect in suppressing monocyte infiltration after ocular trauma (at clinical acceptable doses). D-G: In contrast, TNF- $\alpha$ or IL-1 $\beta$ inhibitor (at clinically acceptable doses) prevents peripheral CX3CR $1^{+}$monocyte infiltration into the retina after surgery (D, E, and $\mathbf{G}$ ), whereas concomitant inhibition of TNF- $\alpha$ and IL-1 $\beta$ has an additive effect, causing further reduction in peripheral monocyte infiltration ( $\mathbf{F}$ and $\mathbf{G})$. G: Analysis of variance analysis with Tukey correction for multiple comparison. $n=4$ mice per group (A-G). ${ }^{\star} P<0.05$,

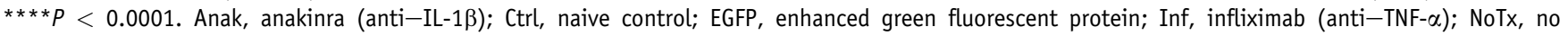
treatment control. 


\section{Quantitative Real-Time PCR}

Eyes were harvested for quantitative real-time PCR at 24 hours after the injury. Corneal and retinal tissues were surgically dissected, placed in cryovials, and frozen in liquid nitrogen. Retinal RNA isolation was performed using RNeasy Mini Kit (74,106; Qiagen, Valencia, CA) and quantified using nanodrop spectrophotometer (Nanodrop 2000; Thermo Scientific, Waltham, MA). Each sample was normalized, and cDNA synthesis was performed using $\mathrm{Su}$ perScript IV VILO (11756050; ThermoFisher, Carlsbad, CA). cDNA ( $1 \mu \mathrm{L})$ was used for RT-PCR. The following TaqMan probes were used: TNFA (Mm99999068_m1; Life Technologies, Woburn, MA), ILIB (MmMm00434228_m; Thermo Scientific), and IL6 (Mm00446190_m1; Thermo Scientific). The average $\mathrm{C}_{\mathrm{T}}$ value was used in the analysis. $\mathrm{C}_{\mathrm{T}}$ values were normalized using $18 S$ (Hs99999901_s1; Thermo Scientific), and ranges were normalized based on naive control tissue. $\Delta \Delta \mathrm{C}_{\mathrm{T}}$ algorithm was used to compare mRNA levels.

\section{TUNEL Labeling and Quantification}

Eyes were enucleated 24 hours after the injury, fixed in $4 \%$ paraformaldehyde, and embedded in optimal compound temperature OCT (Tissue Tek; Sakura, Leiden, the Netherlands). Multiple sagittal cryosections of approximately $10 \mu \mathrm{m}$ in thickness were obtained from the center and periphery of the globe. Tissue sections were transferred to positive charged glass slides (Superfrost Plus; $75 \times 25$ mm; 1-mm thickness; VWR, Radnor, PA) and stored at $-80^{\circ} \mathrm{C}$ for further processing. Terminal deoxynucleotidyl transferase-mediated dUTP nick-end labeling (TUNEL) labeling was performed on tissue sections using the Roche TUNEL kit (12156792910; Roche, Indianapolis, IN), as previously published. ${ }^{20}$ Slides were mounted with DAPI (UltraCruz; sc-24941; Santa Cruz Biotechnology, Dallas, TX) and coverslip, and imaged using epifluorescent microscopy (Leica DM5500 B; Leica, Wetzlar, Germany). The percentage of TUNEL expression in the peripheral retina was calculated as the area ratio of TUNEL/DAPI (\%) using Particle Analysis of ImageJ software version 1.52p (NIH, Bethesda, MD; http://imagej.nih.gov/ij). Quantitative data were presented as means $\pm \mathrm{SD}$.

\section{Cellular Assessment by Flow Cytometry}

Eyes were harvested 7 days after the injury for flow cytometry, and retinas were digested to generate single cells for flow cytometry using collagenase Type I and Papain Dissociation System (Worthington Biochemical Corp., Lakewood, NJ). Allophycocyanin anti-mouse CD45 antibody (clone 1040) and PerCP anti-mouse/human CD11b antibody (clone M1/70) were used. All antibodies were purchased from BioLegend (San Diego, CA). Cells were analyzed on a BD LSR II cytometer (BD Biosciences, San Jose, CA) using FlowJo software version 10.6.2 (Tree Star, Ashland, OR).

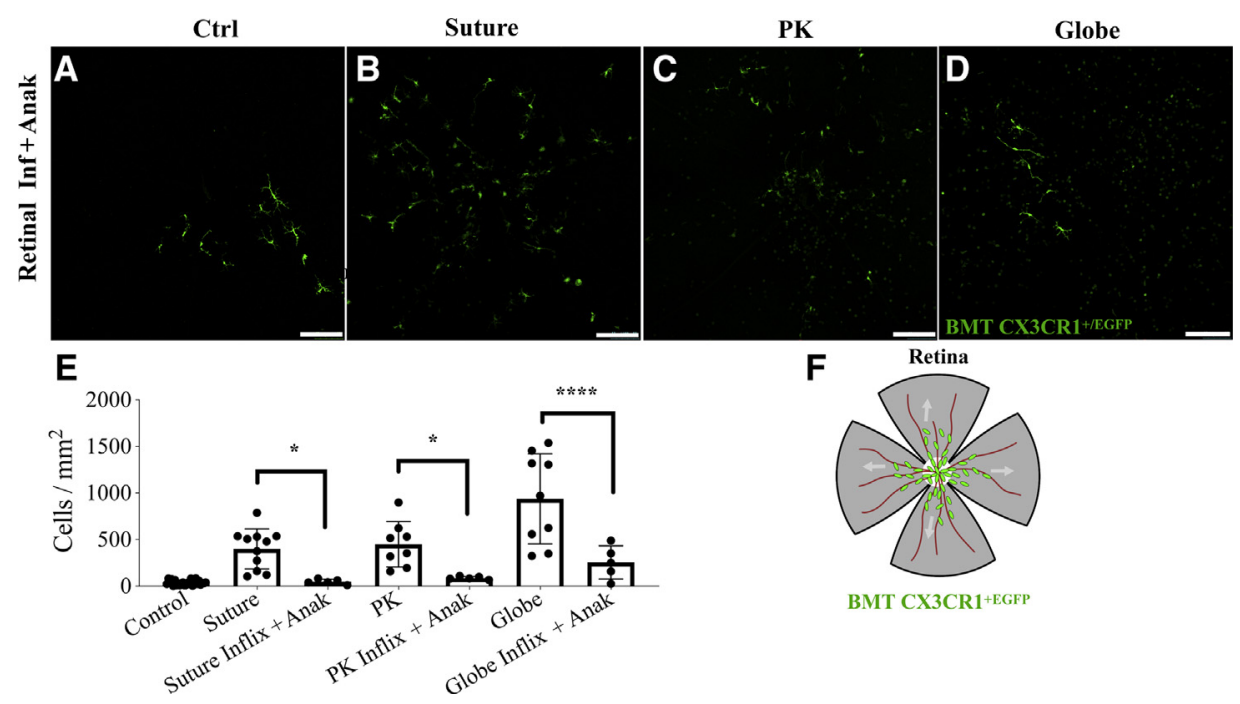

Figure 3 Concomitant inhibition of tumor necrosis factor- $\alpha$ (TNF- $\alpha$ ) and IL-1 $\beta$ suppresses C-X3-C motif chemokine receptor 1-positive (CX3CR1 ${ }^{+}$) monocyte entry into the retina following surgical or globe trauma. A-D: Confocal images of flat mount retinas from BL6; $\mathrm{C} 3 \mathrm{Cr} 1^{+/ E G F P}$ chimeras 24 hours after corneal suture injury, penetrating keratoplasty (PK), and PK with globe (Globe) trauma. E: Quantification of the relative number of CX3CR1 ${ }^{+}$monocyte infiltration into the retina. Concomitant inhibition of TNF- $\alpha$ and IL-1 $\beta$ inhibitors have a significant effect in suppressing peripheral CX3CR1 $1^{+}$monocyte entry into the retina following surgical or globe trauma. After TNF- $\alpha$ and IL-1 $\beta$ inhibition, the number of peripheral CX3CR1 ${ }^{+}$cells in the retina is similar to that of naive mice. F: Schematic representation of flat mount retinas, showing the optic nerve (central white circle), the retinal vessels (red lines), and the infiltrating CX3CR1 ${ }^{+}$monocytes (green oval). E: Analysis of variance analysis with Tukey correction for multiple comparison. $n \geq 8$ mice per group (E). ${ }^{\star} P<0.05,{ }^{* * *} P<0.0001$. Scale bars $=100 \mu \mathrm{m}(\mathbf{A}-\mathbf{D})$. Anak, anakinra (anti-IL-1 $\beta$ ); Ctrl, naive control; EGFP, enhanced green fluorescent protein; Inflix, infliximab (anti-TNF- $\alpha$ ). 

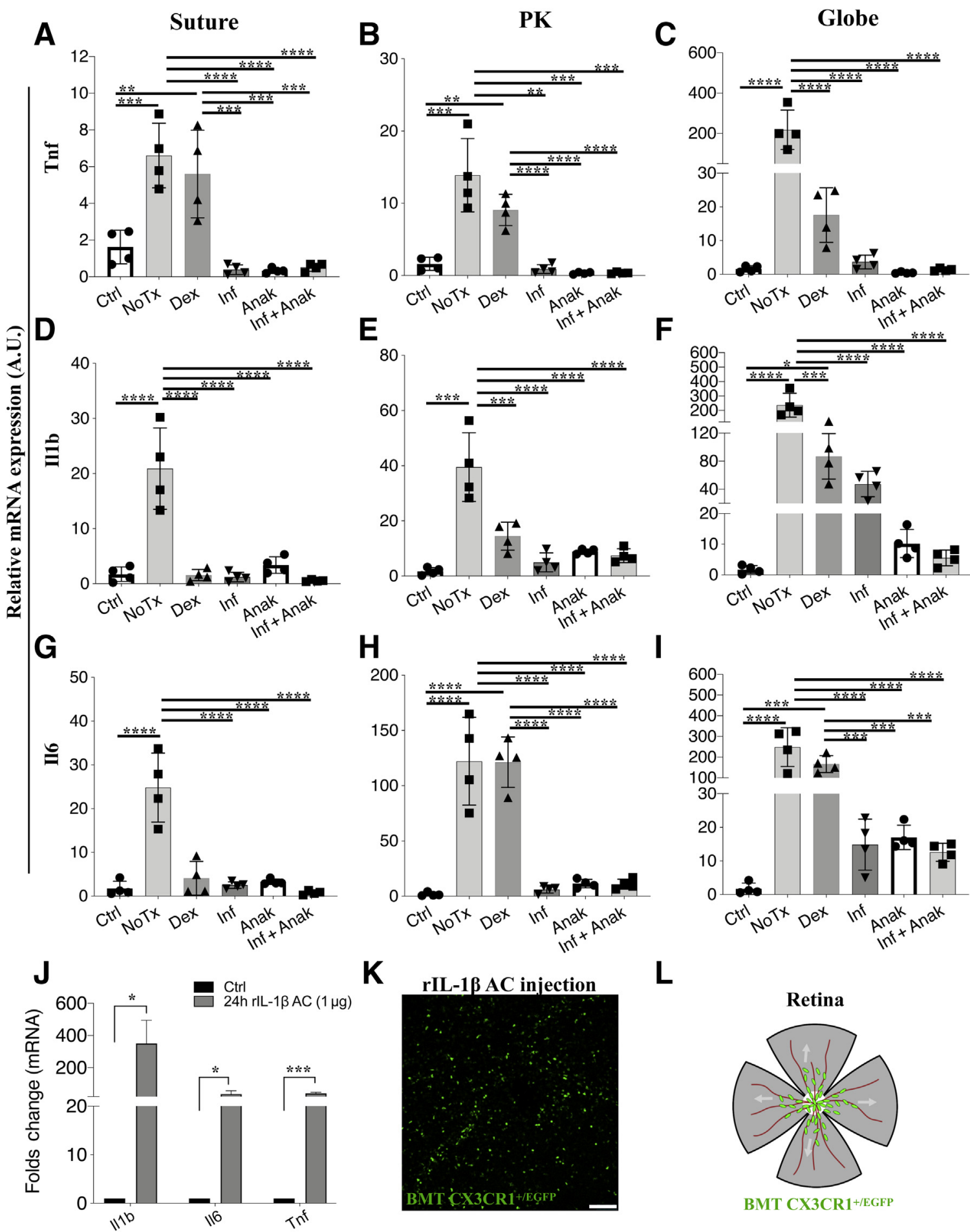

Figure 4 0cular surgical trauma causes prompt up-regulation of key inflammatory mediators in the retina. A-I: Corneal suture, penetrating keratoplasty (PK), and more severe globe trauma cause tumor necrosis factor- $\alpha$ (Tnf; A-C), Il1b (D-F), and Il6 (G-I) mRNA up-regulation in the retina within 24 hours. The magnitude of this up-regulation depends on the severity of the injury, with globe trauma causing the strongest response, whereas the corneal suture was the weakest. Prompt inhibition of TNF- $\alpha$ or IL-1 $\beta$ after the injury suppresses the inflammatory expression in the retina; however, concomitant TNF- $\alpha$ and IL-1 $\beta$ inhibition is synergistic in Il1b suppression after severe globe trauma. In contrast, systemic administration of dexamethasone (Dex) has limited effect in suppression of retinal inflammation and is therapeutically inferior to TNF- $\alpha$ or IL-1 $\beta$ inhibitors. J and K: Injection of recombinant IL-1 $\beta$ ( $1 \mu \mathrm{g}$ in $1 \mu \mathrm{L}$ ) in the anterior chamber causes significant up-regulation of Il1b, Tnf, and Il6 mRNA (J) and infiltration of bone marrow-derived C-X3-C motif chemokine receptor 1-positive (CX3CR1 $\left.{ }^{+/ E G F P}\right)$ monocytes/macrophages in the retina within 24 hours (K). A-I: Analysis of variance analysis with Tukey correction for multiple comparison. J: Independent $t$-test. L: Schematic representation of flat mount retinas, showing the optic nerve (central white circle), the retinal vessels (red lines), and the infiltrating CX3CR1 ${ }^{+}$monocytes (green oval). $n=4 \mathrm{C} 57 \mathrm{BL} / 6 \mathrm{mice}$ per group $(\mathbf{A}-\mathbf{J}) .{ }^{*} P<0.05,{ }^{* *} P<0.01,{ }^{* * *} P<0.001$, and ${ }^{* * * * P}<0.0001$. Scale bar $=100 \mu \mathrm{m}$ (K). Anak, anakinra (anti-IL-1 $\beta$ ); A.U., arbitrary unit; Ctrl, naive control; EGFP, enhanced green fluorescent protein; Inf, infliximab (anti-TNF- $\alpha$ ); NoTx, no treatment control; rIL-1 $\beta$, recombinant interleukin 1 beta. 


\section{Flat Mount Retinal Imaging}

Eye were enucleated 24 hours after the injury and fixed in $4 \%$ paraformaldehyde solution for 1 hour at $4{ }^{\circ} \mathrm{C}$. Cornea and retina tissues were carefully dissected, washed three times for 5 minutes in phosphate-buffered saline solution at $4^{\circ} \mathrm{C}$, and mounted using VECTRASHIELD mounting medium (H-1000; Vector Laboratories, Burlingame, CA) and a coverslip. Confocal images were taken using the Leica SP8 confocal microscope.

\section{Statistical Analysis}

The normality of continuous variables was assessed using the Kolmogorov-Smirnov test. ${ }^{36}$ Quantitative variables were expressed as means $\pm \mathrm{SD}$, and qualitative variables were expressed as frequencies and percentages. Multiple comparison tests were performed using one-way analysis of variance, with Tukey correction. All tests were two tailed, and statistical significance was determined at $P<0.05$. Results were analyzed with Prism version 8.2.1 (GraphPad Software Inc., San Diego, CA).

\section{Results}

Ocular Surgery or Trauma Causes Monocyte Infiltration into the Retina within 24 Hours, Introducing a New Pathway to Further Neuroretinal Degeneration

CX3CR1 is a chemokine receptor expressed primarily by bone marrow-derived macrophages and microglia. ${ }^{32}$ The effect of ocular injury (surgical or trauma) on retinal innate immune response was evaluated using C57BL/6 mice transferred with CX3CR1 ${ }^{+/ E G F P}$ bone marrow cells following busulfan myelodepletion (Figure 1, A-D). It has been previously shown that busulfan myelodepletion does not cause blood retinal barrier disruption, and hence, peripheral monocyte infiltration into the retina would be the result of experimental injury. ${ }^{31-33}$

Herein, it is shown that all types of ocular injuries [corneal suture, penetrating keratoplasty (PK), and globe trauma]

TUNEL 24h PK

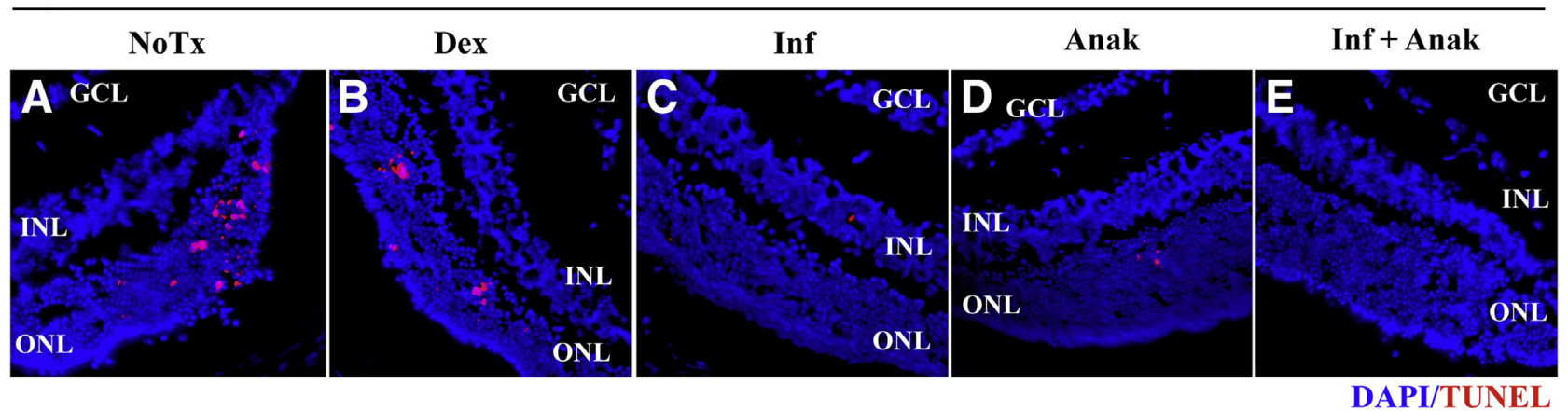

$\mathbf{F}$

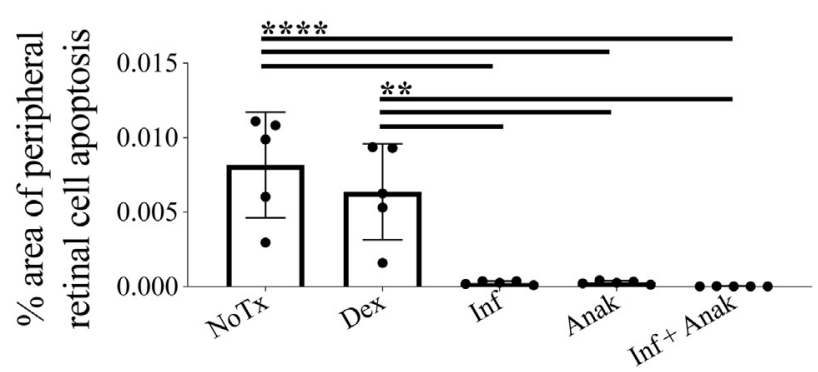

$\mathbf{G}$

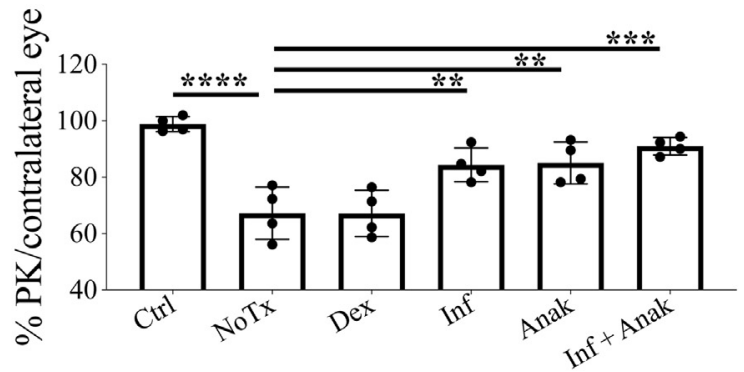

Figure 5 Tumor necrosis factor- $\alpha$ (TNF- $\alpha$ ) and IL-1 $\beta$ inhibition prevents retinal damage and ganglion cell loss after ocular surgical trauma. A-E: Retinal cell apoptosis 24 hours after penetrating keratoplasty (PK), using terminal deoxynucleotidyl transferase-mediated dUTP nick-end labeling (TUNEL) assay (apoptotic cells: red). F: Quantification of relative cell apoptosis using pixel density analysis. G: Quantification of retinal Thy- 1 cell surface antigen (Thy ${ }^{+}$) ganglion cell number at 7 days using flow cytometry. A and F: PK injury causes retinal cell apoptosis within 24 hours of surgery, especially in the peripheral retina. B and F: Systemic administration of dexamethasone (Dex) has limited effect on retinal protection. C-F: In contrast, systemic administration of TNF- $\alpha$ or IL-1 $\beta$ inhibitors abrogates retinal cell apoptosis, whereas concomitant inhibition of TNF- $\alpha$ and IL-1 $\beta$ provides similar protection of the retinal cells as monotherapy. G: At 7 days after the injury, the number of Thy $1^{+}$retinal neuron cells exhibits a significant reduction. This loss of Thy $1^{+}$cells cannot be inhibited by dexamethasone administration (at clinically acceptable doses), but can be prevented using TNF- $\alpha$ or IL-1 $\beta$ inhibitors (at clinically acceptable doses). Concomitant TNF- $\alpha$ and IL-1 $\beta$ inhibition confers similar protection to $\mathrm{Thy}^{+}{ }^{+}$cells as monotherapy. F and G: Analysis of variance analysis with Tukey correction for multiple comparison. $n=5 \mathrm{C} 57 \mathrm{BL} / 6$ mice per group (F); $n=4$ Thy $1^{\mathrm{YFP} / \mathrm{FFP}}$ mice per group (G). ${ }^{* *} P<0.01,{ }^{* *} P<0.001$, and ${ }^{* * * *} P<0.0001$. Images obtained with a $25 \times$ objective $(\mathbf{A}-\mathbf{E})$. Anak, anakinra (anti-IL-1 $\left.\beta\right) ;$ Ctrl, naive control; $\mathrm{GCL}$, ganglion cell layer; Inf, infliximab (anti-TNF- $\alpha$ ); INL, inner nuclear layer; ONL, outer nuclear layer; NoTx, no treatment control; YFP, yellow fluorescent protein. 
cause significant infiltration of $\mathrm{CX} \mathrm{CR} 1^{+}$bone marrow-derived monocytes into the retina within 24 hours (Figure 1, E-J). The density of infiltrated cells is highest around the optic nerve head (Figure 1, E-H). Globe trauma caused significantly more peripheral $\mathrm{CX} 3 \mathrm{CR} 1^{+}$monocyte infiltration into the retina, as opposed to corneal suture injury, which caused the least (Figure 1I). Schematic representation of flat mount retinal images is given (Figure $1 \mathrm{~J}$ ).

Further analysis was performed using flow cytometry of Cx3 $\mathrm{CrI}^{+/ \text {EGFP }}$ reporter mice after PK injury. At 24 hours after the injury, the retina exhibited significant increase in $\mathrm{CX} 3 \mathrm{CR} 1^{+} \mathrm{CD} 5^{+}$monocytes. This increase was attributed to $\mathrm{CD} 45^{\text {hi }}$ bone marrow-derived monocyte infiltration rather that $\mathrm{CD} 45^{\text {lo }}$ yolk sac-derived microglia proliferation. Further phenotype analysis of the cells showed that most (75\%) of the CX3CR $1^{+}$cells had peripheral origin $\left(\mathrm{CX} 3 \mathrm{CR} 1^{\mathrm{lo}}\right)$ as opposed to naive mice, where $100 \%$ of the monocytes were CX3CR $1^{\text {hi }}$ yolk sac-derived microglia (Figure $1 \mathrm{~K}$ ).

\section{TNF- $\alpha$ or IL-1 $\beta$ Inhibition Prevents Peripheral CX3CR1 ${ }^{+}$ Monocyte Infiltration into the Retina}

It was previously shown that infiltration of peripheral monocytes into the retina is a major mediator of long-term retinal inflammation and degeneration following chemical insult. ${ }^{32,33}$ Conversely, preventing these cells from entering into the retina is neuroprotective. ${ }^{32,33}$ Herein, systemic administration of TNF- $\alpha$ or IL-1 $\beta$ inhibitor prevented peripheral CX3CR $1^{+}$monocyte infiltration into the retina after PK. Concomitant inhibition of TNF- $\alpha$ and IL-1 $\beta$ had an additive effect, causing further reduction in peripheral monocyte infiltration (Figure 2). In contrast, systemic administration of corticosteroids (dexamethasone) had only a minimal effect and was significantly inferior to TNF- $\alpha$ or IL-1 $\beta$ inhibition in suppressing monocyte infiltration after ocular trauma (Figure 2).

Further investigation of the effect of combined TNF- $\alpha$ and IL-1 $\beta$ inhibition on peripheral immune cells was performed in C57BL/6 mice transferred with CX3CR1 $1^{+/ E G F P}$ bone marrow cells. Concomitant inhibition of anti-TNF- $\alpha$ and IL- $1 \beta$ significantly suppressed CX3CR $1^{+}$monocyte entry into the retina following surgical or globe trauma (Figure 3, A-E). After TNF- $\alpha$ and IL-1 $\beta$ inhibition, the number of peripheral $\mathrm{CX} 3 \mathrm{CR} 1^{+}$cells in the retina was similar to that of naive mice (Figure 3E). Schematic representation of flat mount retinal images is given (Figure 3F).

Surgical Injury and Globe Trauma Cause Rapid Retinal Inflammation and Cell Apoptosis that Can Be Ameliorated by TNF- $\alpha$ and IL-1 $\beta$ Inhibitors

It has been previously shown that ocular chemical trauma and corneal surgery cause increased $T N F-\alpha$ and $I L-1 \beta$ expression in the retina. ${ }^{18,19,21}$ This study expands on those findings to show that corneal suture, $\mathrm{PK}$, and more severe globe trauma all cause Tnf, Illb, and Il6 mRNA up-regulation in the retina within 24 hours (Figure 4, A-I). The magnitude of this upregulation depended on the severity of the injury, with globe trauma causing the strongest response, and corneal suture the weakest (Figure 4, A-I). Prompt inhibition of TNF- $\alpha$ or IL-1 $\beta$ after the injury suppressed inflammatory gene expression in the retina (Figure 4, A-I), and concomitant TNF- $\alpha$ and IL-1 $\beta$ inhibition was better in suppressing retinal Illb expression after severe globe trauma (Figure 4F). In contrast, systemic administration of dexamethasone had a limited effect in suppression of retinal cytokine expression, and was therapeutically inferior to TNF- $\alpha$ or IL- $1 \beta$ inhibitors (Figure $4, \mathrm{~A}-\mathrm{I}$ ). To assess the role of cytokine diffusion from the injured cornea back into the retina and its effect on retinal inflammation and monocyte infiltration, anterior chamber injection was performed with recombinant IL-1 $\beta$. At 24 hours after the injection, the retina exhibited significant up-regulation of Il1b, Il6, and Tnf mRNA levels (Figure 4J) and marked infiltration of bone marrow-derived CX3CR $1^{+/ \text {EGFP }}$ cell (Figure $4 \mathrm{~K}$ ). Schematic representation of flat mount retinal images is given (Figure 4L).

Retinal cell apoptosis was previously identified as an early marker of retinal damage after chemical injury. ${ }^{18,20}$ In the current study, PK also causes retinal cell apoptosis at 24 hours of surgery, especially in the peripheral retina. This was abrogated with prompt administration of TNF- $\alpha$ or IL- $1 \beta$ inhibitors (Figure 5, A and F). In contrast, administration of dexamethasone had a limited protective effect and was therapeutically inferior in retinal protection compared with TNF- $\alpha$ or IL-1 $\beta$ inhibitors (Figure 5, B and F). Concomitant inhibition of anti-TNF- $\alpha$ and IL- $1 \beta$ provided similar protection to the retinal cells as monotherapy (Figure 5, C-F). Thy 1 is a marker of retinal neuron cells, used for the quantification of rental ganglion cells. ${ }^{37}$ At 7 days after PK, the number of Thy $1^{+}$ retinal ganglion cells was significantly reduced. This loss of Thy $1^{+}$cells was not inhibited by dexamethasone administration $(20 \mathrm{mg} / \mathrm{kg})$, but was prevented using TNF- $\alpha$ or IL-1 $\beta$ inhibition (Figure 5G). Concomitant TNF- $\alpha$ and IL-1 $\beta$ inhibition conferred protection of Thy $1^{+}$cells similar to either agent alone (Figure 5G).

\section{Discussion}

Herein, we first confirm and expand on previous findings that mechanical trauma or ocular surgery can cause retinal damage, including ganglion cell apoptosis, through an inflammatory mechanism. ${ }^{18-20,38}$ To this end, three different ocular injury models (namely, a full-thickness corneal suture, PK, and PK with globe trauma) were employed to show that all injuries, even a single corneal suture, can cause not only rapid neuroglia changes in the retina but also retinal ganglion cell death.

Thus, there is robust evidence for the concept of TNF- $\alpha$, IL- $1 \beta$, and other cytokines causing direct damage to the ganglion cells, but not necessarily mediated by IOP increase. ${ }^{22}$ Such ganglion cell attrition is known to lead to 
glaucoma. ${ }^{39}$ Anti-TNF- $\alpha$ monoclonal antibodies, such as infliximab or adalimumab, are markedly effective in retinal ganglion cell protection and can therefore be expected to prevent the time bomb of glaucoma. ${ }^{18,20,21,38}$ These findings may lead to effective prophylaxis against glaucoma after trauma or surgery.

However, recent discoveries suggest that there may be additional pathways to the ganglion cell attrition and delayed glaucoma due to trauma. ${ }^{20}$ Monocytes/macrophages rapidly infiltrate the retina after eye trauma and by TNF- $\alpha$ secretion or other mechanism prolong and perpetuate the retinal damage. This new potential pathway to glaucoma has been the focus of the present study. Thus, CX3CR $1^{+}$monocytes were found to infiltrate the retina of mice within 24 hours after the trauma, thereby increasing the number of $\mathrm{CD} 45^{+}$myeloid cells in the retina. Because the retina is populated exclusively by nonperipheral, yolk sac-derived microglia, ${ }^{30,40}$ the presence of peripheral monocytes is deemed pathologic i $^{32,34,41}$ and likely contributes to neurodegeneration, as previously shown in chemical burns. ${ }^{31-33}$ Although TNF- $\alpha$ released from the injured cornea of mice ceases within weeks of the injury, ${ }^{20}$ local retinal TNF- $\alpha$ secretion from engrafted monocytes continues for years, ${ }^{31}$ and is thereby expected to contribute to an autonomous, long-lasting degeneration of the retina. Trigeminal nerve stimulation from the injured cornea may also contribute to the neuroinflammatory signal to the retina, as previously shown for the brain. ${ }^{42}$ In this study, we provide compelling evidence to support that cytokine diffusion from the injured cornea to the retina is the most likely signaling mechanism of neuroretinal inflammation and monocyte infiltration; injection of recombinant IL-1 $\beta$ in the anterior chamber causes similar inflammatory response and monocyte recruitment by the retina as in corneal surgical or traumatic injury. The detrimental role of monocyte infiltration into the retina was also confirmed in a light-inducible mouse model of photoreceptor degeneration, ${ }^{34}$ corroborating earlier findings that engrafted monocytes are involved in long-term neurodegeneration of the retina. ${ }^{31,33}$

More important, it is shown that blockade of TNF- $\alpha$ or IL-1 $\beta$ prevents $\mathrm{CX} 3 \mathrm{CR} 1^{+}$monocyte infiltration into the retina. Somewhat surprisingly, systemic dexamethasone had minimal effect. The limited immunosuppressive effect of dexamethasone, especially in severe ocular injuries, is consistent with previous observations ${ }^{20}$ and underlines the therapeutic importance of targeted immunomodulation over steroids. This is likely attributed to other molecules, that are simultaneously released by the injured tissue, such as interferon- $\gamma$, which can inhibit the function of glucocorticosteroids. ${ }^{43}$ This has practical implications for future efforts to prevent glaucoma after ocular trauma and suggests that systemic dexamethasone, although it reduces clinically evident anterior segment inflammation, does not provide adequate protection of the retinal ganglion cells. Antibodies targeting inflammatory cytokines seem to work well on multiple pathways against glaucoma. ${ }^{22}$
Regardless of the mechanism of damage to the retinal ganglion cells, whether triggered by IOP increase or directly effected by cytokines, or indirectly via invading monocytes, prophylaxis with anti-TNF- $\alpha$ or IL- $1 \beta$ antibodies appeared in the current study models to result in significant protection.

The effect of TNF- $\alpha$ inhibition on Il1b mRNA expression has been previously reported ${ }^{20,44}$ and is likely mediated by signaling cross talk and effects on the induction of IL-1 $\beta$ rather than on the secretion or processing. Monocytes/ macrophages/microglia secrete TNF- $\alpha$, IL- $1 \beta$, and IL- 6 , and these cytokines share common intracellular signaling pathways, such as STAT3 ${ }^{45}$ Neutralization of one of the proteins can therefore affect downstream signals through STAT3 and ultimately alter the phenotype of the cell. All these changes are expected to affect the regulation of multiple genes through autocrine/paracrine signaling. ${ }^{44}$

Acute IOP elevation is still considered the major risk factor for post-traumatic glaucoma, and maximal IOP lowering therapy should be given, as outlined in earlier work. ${ }^{17}$ IOP levels were not assessed in this study, but the possible impact of IOP elevation in the mouse eye after corneal suture, $\mathrm{PK}$, or globe trauma was negated by prompt treatment with either anti-TNF- $\alpha$ or anti-IL-1 $\beta$. Further studies are needed to assess the relationship between IOP and inflammation in the pathogenesis of post-traumatic glaucoma.

Although in traumatic ocular injuries, the above described events cannot be prevented, prompt intervention with antibodies may minimize the damage, suppress inflammation, and protect the remaining neurons. In elective procedures, however, such as PK or cataract, pre-emptive administration of inhibitors may prevent these events, protect the neurons, and minimize the known post-surgery complication of glaucoma. Future clinical studies will help assess the potential benefits of such therapy.

In conclusion, acute globe trauma, whether accidental or due to surgery, can cause indirect damage to the retina and result in glaucoma through release of inflammatory cytokines. Monocyte infiltration into the retina after an injury as minimal as corneal suture suggests previously unappreciated links between the anterior and posterior eye, mediated by the expression of inflammatory mediators. Both inflammatory gene expression and monocyte infiltration were prevented by TNF- $\alpha$ or IL-1 $\beta$ inhibitors. Thus, targeted immunomodulation with TNF- $\alpha$ or IL- $1 \beta$ blockade, combined with IOP reduction, deserves further study in the prevention of post-traumatic glaucoma.

\section{Author Contributions}

X.C., F.L., and C.Z. performed experiments, acquired, and analyzed data; J.C., L.W., and Y.H. critically reviewed the manuscript; C.H.D. and E.I.P. designed experiments and wrote the manuscript. 


\section{References}

1. Campbell DG: Traumatic glaucoma. Edited by Shingleton BJ, Hersh PS, Kenyon KR. In Eye Trauma. St. Louis, MO: Mosby-Year Book, 1991. pp. 117-125

2. Tsai JH, Derby E, Holland EJ, Khatana AK: Incidence and prevalence of glaucoma in severe ocular surface disease. Cornea 2006, 25: $530-532$

3. Pfister RR, Pfister DR: Alkali injuries of the eye. Edited by Krachmer JH, Mannis MJ, Holland EJ. In Cornea. Vol I, ed 3. Elsevier, 2011. pp. 1193-1202

4. Kuckelkorn R, Keller GK, Redbrake C: Glaucoma after extremely severe chemical and thermal eye burns: surgical possibilities. Ophthalmologe 2001, 98:1149-1156

5. Ayala RS: Penetrating keratoplasty and glaucoma. Surv Ophthalmol 2000, 45:91-105

6. Rumelt S, Bersudsky V, Blum-Hareuveni T, Rehany U: Preexisting and postoperative glaucoma in repeated corneal transplantation. Cornea 2002, 21:759-765

7. Al-Mahmood AM, Al-Swailem SA, Edward DP: Glaucoma and corneal transplant procedures. J Ophthalmol 2012, 2012:576394

8. Haddadin RI, Chodosh J: Corneal transplantation and glaucoma. Semin Ophthalmol 2014, 29:380-386

9. Wu S, Xu J: Incidence and risk factors for post-penetrating keratoplasty glaucoma: a systemic review and meta-analysis. PLoS One 2017, 12:e0176261

10. Netland PA, Terada H, Dohlman $\mathrm{CH}$ : Glaucoma associated with keratoprosthesis. Ophthalmology 1998, 105:751-757

11. Harissi-Dagher M, Dohlman CH: The Boston keratoprosthesis in ocular trauma. Can J Ophthalmol 2008, 43:165-169

12. Cade F, Grosskreutz CL, Tauber A, Dohlman CH: Glaucoma in eyes with severe chemical burn, before and after keratoprosthesis. Cornea 2011, 30:1322-1327

13. Crnej A, Paschalis EI, Salvador-Culla B, Tauber A, DrnovsekOlup B, Shen LQ, Dohlman CH: Glaucoma progression and role of glaucoma surgery in patients with Boston keratoprosthesis. Cornea 2014, 33:349-354

14. Kamyar R, Weizer JS, de Paula FH, Stein JD, Moroi SE, John D, Musch DC, Mian SI: Glaucoma associated with Boston type I keratoprosthesis. Cornea 2012, 31:134-139

15. Talajic JC, Agoumi Y, Gagne S, Moussally K, Harissi-Dagher M: Prevalence, progression, and impact of glaucoma on vision after Boston type I keratoprosthesis surgery. Am J Ophthalmol 2012, 153:267-274.e1

16. Iyer G, Srinivasan B, Agarwal B, Shetty R, Krishnamoorthy S, Balekudaru S, Vijaya L: Glaucoma in modified osteo-odontokeratoprosthesis eyes: role of additional stage IA and Ahmed glaucoma drainage device-techniques. Am J Ophthalmol 2015, 159:482-489

17. Dohlman CH, Robert MC, Paschalis EI: Treatment of chemical burns to the eye: a changing picture. Edited by Colby K, Dana MR. In Foundation of Corneal Disease. Denver, CO: Springer, 2020. pp. 109-119

18. Cade F, Paschalis EI, Regatieri CV, Vavvas DG, Dana R, Dohlman CH: Alkali burn to the eye: protection using TNF- $\alpha$ inhibition. Cornea 2014, 33:382-389

19. Crnej A, Omoto M, Dohlman TH, Gonzalez-Andrades M, Paschalis EI, Cruzat A, Vu TH, Doorenbos M, Chen DF, Dohlman CH, Dana R: Effect of penetrating keratoplasty and keratoprosthesis implantation on the posterior segment of the eye. Invest Ophthalmol Vis Sci 2016, 57:1643-1648

20. Paschalis EI, Zhou C, Lei F, Scott N, Kapoulea V, Robert MC, Vavvas D, Dana R, Chodosh J, Dohlman CH: Mechanisms of retinal damage after ocular alkali burns. Am J Pathol 2017, 187:1327-1342

21. Dohlman CH, Cade F, Regatieri CV, Zhou C, Lei F, Crnej A, HarissiDagher M, Robert MC, Papaliodis GN, Chen D, Aquavella JV, Akpek EK, Aldave AJ, Sippel KC, D’Amico DJ, Dohlman JG, Fagerholm P, Wang L, Shen LQ, González-Andrades M, Chodosh J, Kenyon KR, Foster CS, Pineda R, Melki S, Colby KA, Ciolino JB,
Vavvas DG, Kinoshita S, Dana R, Paschalis EI: Chemical burns of the eye: the role of retinal injury and new therapeutic possibilities. Cornea 2018, 37:248-251

22. Dohlman CH, Zhou C, Lei F, Cade F, Regatieri CV, Črnej A, Dohlman JG, Shen LQ, Paschalis EI: Glaucoma after corneal trauma and surgery - a rapid, inflammatory, IOP-independent pathway. Cornea 2019, 38:1589-1594

23. Sotozono C, He J, Matsumoto Y, Kita M, Imanishi J, Kinoshita S: Cytokine expression in the alkali-burned cornea. Curr Eye Res 1997, 16:670-676

24. Miyamoto F, Sotozono C, Ikeda T, Kinoshita S: Retinal cytokine response in mouse alkali-burned eye. Ophthalmic Res 1998, 30:168-171

25. Yamada J, Dana MR, Sotozono C, Kinoshita S: Local suppression of IL-1 by receptor antagonist in the rat model of corneal alkali injury. Exp Eye Res 2003, 76:161-167

26. Nakazawa T, Nakazawa C, Matsubara A, Noda K, Hisatomi T, She H, Michaud N, Hafezi-Moghadam A, Miller JW, Benowitz LI: Tumor necrosis factor-alpha mediates oligodendrocyte death and delayed retinal ganglion cell loss in a mouse model of glaucoma. J Neurosci 2006, 26:12633-12641

27. Roh M, Zhang Y, Murakami Y, Thanos A, Lee SC, Vavvas DG, Benowitz LI, Miller JW: Etanercept, a widely used inhibitor of tumor necrosis factor- $\alpha$ (TNF- $\alpha$ ), prevents retinal ganglion cell loss in a rat model of glaucoma. PLoS One 2012, 7:e40065

28. Choi H, Phillips C, Oh JY, Stock EM, Kim DK, Won JK, Fulcher S: Comprehensive modeling of corneal alkali injury in the rat eye. Curr Eye Res 2017, 42:1348-1357

29. Paschalis EI, Taniguchi EV, Chodosh J, Pasquale LR, Colby K, Dohlman CH, Shen LQ: Blood levels of tumor necrosis factor alpha and its type 2 receptor are elevated to patients with Boston type 1 keratoprosthesis. Curr Eye Res 2019, 44:599-606

30. Mildner A, Schmidt H, Nitsche M, Merkler D, Hanisch UK, Mack M, Heikenwalder M, Brück W, Priller J, Prinz M: Microglia in the adult brain arise from Ly-6ChiCCR2+ monocytes only under defined host conditions. Nat Neurosci 2007, 10:1544-1553

31. Paschalis EI, Lei F, Zhou C, Kapoulea V, Dana R, Chodosh J, Vavvas DG, Dohlman CH: Permanent neuroglial remodeling of the retina following infiltration of CSF1R inhibition-resistant peripheral monocytes. Proc Natl Acad Sci USA 2018, 115:E11359-E11368

32. Paschalis EI, Lei F, Zhou C, Kapoulea V, Thanos A, Dana R, Vavvas DG, Chodosh J, Dohlman CH: The role of microglia and peripheral monocytes in retinal damage after corneal chemical injury. Am J Pathol 2018, 188:1580-1596

33. Paschalis EI, Zhou C, Chen XN, Kapoulea V, Hui PC, Dana R, Chodosh J, Vavvas DG, Dohlman CH: Microglia regulate neuroglia remodeling in various ocular and retinal injuries. J Immunol 2019, 202: 539-549

34. Karlen SJ, Miller EB, Wang X, Levine ES, Zawadzki RJ, Burns ME: Monocyte infiltration rather than microglia proliferation dominates the early immune response to rapid photoreceptor degeneration. J Neuroinflammation 2018, 15:344

35. Committee for the Update of the Guide for the Care and Use of Laboratory Animals; National Research Council: Guide for the Care and Use of Laboratory Animals: Eighth Edition. Washington, DC, National Academies Press, 2011

36. Dodge Y: The Concise Encyclopedia of Statistics. Washington, DC: Springer Science \& Business Media, 2008

37. Groman-Lupa S, Adewumi J, Park KU, Brzezinski JI: The transcription factor Prdm16 marks a single retinal ganglion cell subtype in the mouse retina. Invest Ophthalmol Vis Sci 2017, 58:5421-5433

38. Zhou C, Robert MC, Kapoulea V, Lei F, Stagner AM, Jakobiec FA, Dohlman CH, Paschalis EI: Sustained subconjunctival delivery of infliximab protects the cornea and retina following alkali burn to the eye. Invest Ophthalmol Vis Sci 2017, 58:96-105

39. Quigley HA, Dunkelberger GK, Green WR: Retinal ganglion cell atrophy correlated with automated perimetry in human eyes with glaucoma. Am J Ophthalmol 1989, 107:453-464 
40. Hoeffel G, Chen J, Lavin Y, Low D, Almeida FF, See P, Beaudin AE, Lum J, Low I, Forsberg EC, Poidinger M, Zolezzi F, Larbi A, Ng LG, Chan JK, Greter M, Becher B, Samokhvalov IM, Merad M, Ginhoux F: $\mathrm{C}-\mathrm{Myb}(+)$ erythro-myeloid progenitor-derived fetal monocytes give rise to adult tissue-resident macrophages. Immunity 2015, 42:665-678

41. Kettenman H, Hanisch UK, Noda M, Verkhratsky A: Physiology of microglia. Physiol Rev 2011, 91:461-553

42. Ferrari G, Bignami F, Giacomini C, Capitolo E, Comi G, Chaabane L, Rama P: Ocular surface injury induces inflammation in the brain: in vivo and ex vivo evidence of a corneal-trigeminal axis. Invest Ophthalmol Vis Sci 2014, 55:6289-6300
43. Luedke CE, Cerami A: Interferon-gamma overcomes glucocorticoid suppression of cachectin/tumor necrosis factor biosynthesis by murine macrophages. J Clin Invest 1990, 86:1234-1240

44. Mori T, Miyamoto T, Yoshida H, Asakawa M, Kawasumi M, Kobayashi T, Morioka H, Chiba K, Toyama Y, Yoshimura A: IL1beta and TNFalpha-initiated IL-6-STAT3 pathway is critical in mediating inflammatory cytokines and RANKL expression in inflammatory arthritis. Int Immunol 2011, 23:701-712

45. Brennan FM, Chantry D, Jackson A, Maini R, Feldmann M: Inhibitory effect of TNF alpha antibodies on synovial cell interleukin-1 production in rheumatoid arthritis. Lancet 1989, 2:244-247 